\title{
Fusion of Radar and IRST Sensor Measurements for 3D Target Tracking using Extended Kalman Filter
}

\author{
V.P.S. Naidu \\ National Aerospace Laboratories, Bangalore-17, INDIA
}

\begin{abstract}
Tracking algorithms for IRST and radar are implemented and their performance is checked with simulated data. Detailed mathematical expressions given could be useful for implementation. Performance evaluation metrics have been presented to check the tracking algorithm performance. Two fusion schemes have been presented and their performances evaluated with simulated data. It is concluded that both fusion schemes performed alike with the second fusion scheme giving slightly better results. From the results, it is also concluded that fusion of IRST and radar would improve the tracking performance and reduce the positional uncertainty compared to individual trackers.
\end{abstract}

Keywords: IRST sensor measurements, 3-D target tracking, Kalman filter, tracking algorithm, performance evaluation, fusion schemes, target tracking

\section{INTRODUCTION}

Modern military aircraft are equipped with diversity of tracking sensors to guide the pilot. If these sensors are perfect, then the target tracking could be achieved by simple geometry. In general, sensors are not perfect and their measurements are corrupted due to noise. Moreover, single sensor may not provide all information about the target. Hence, filters and multi-sensors are used to enhance the target-tracking capabilities. Radar can measure azimuth, elevation, and range of the target. It can measure range with good resolution, but the angular measurements are not with good resolution. Radar provide sufficient information to track the target since it measures both angular position and range of the target. The uncertainty associated with radar might be represented as a volume whose dimensions are relatively large perpendicular to the measured line-ofsight and small along the line-of-sight. An infrared search and track (IRST) sensor (sometimes known as infrared sighting and tracking) can measure azimuth and elevation of a target with good resolution. It can provide only the direction of the target but not its location because it does not provide the range. The uncertainty associated with IRST might be represented as a square whose dimensions are comparatively small perpendicular to the measured lineof-sight. An improved estimate of target location and reduced positional uncertainty would result from the fusion of information obtained from multi sources ${ }^{1-3}$. With the fusion of measurements from radar and IRST, the resultant uncertainty of the estimated position of the target is smaller than the uncertainty of the estimates with either measurement alone.

This paper deals with tracking of target in 3-D Cartesian coordinates using IRST and radar measurements. The information flow diagram is shown in Fig. 1. Extended Kalman filter is used to estimate the state of a target using target motion and measurement models. Mathematical models for radar and IRST sensors, extended Kalman filter for tracking, and two track-to-track fusion schemes are presented. The performance of the tracking algorithm is presented in terms of percentage fit error (PFE), root mean square error in position, velocity and acceleration, root sum square error in position, mean absolute error in position, and innovation sequence with theoretical bounds etc.

\section{SENSOR AND TARGET MOTION MODELS}

For simplicity it is assumed that sampling rate of both radar and IRST is the same. The dynamics of the target can be represented as

$$
X(k)=F X(k-1)+G w(k-1)
$$

The measurements corresponding to the radar and IRST are

$$
z_{m}^{n}(k)=h^{n}(X(k))+v^{n}(k)
$$

where $X(k)$ is the state vector, $F$ is the state transition matrix, and $G$ is the process noise gain matrix. The process noise $w(k)$ and the measurement noise $v^{n}(k)$ are zero-mean, mutually independent, white, Gaussian with covariance $Q$ and $R^{n}$ respectively. $z_{m}^{n}(k)$ is the measurement of the sensor $n$ at time $k$ and the observation matrix $h^{n}(X(k))$ is a nonlinear function of the states computed at time $k$. The index $k$ indicates the sample/scan number.

\subsection{Sensor Model}

In most of the aerospace applications, sensors are 


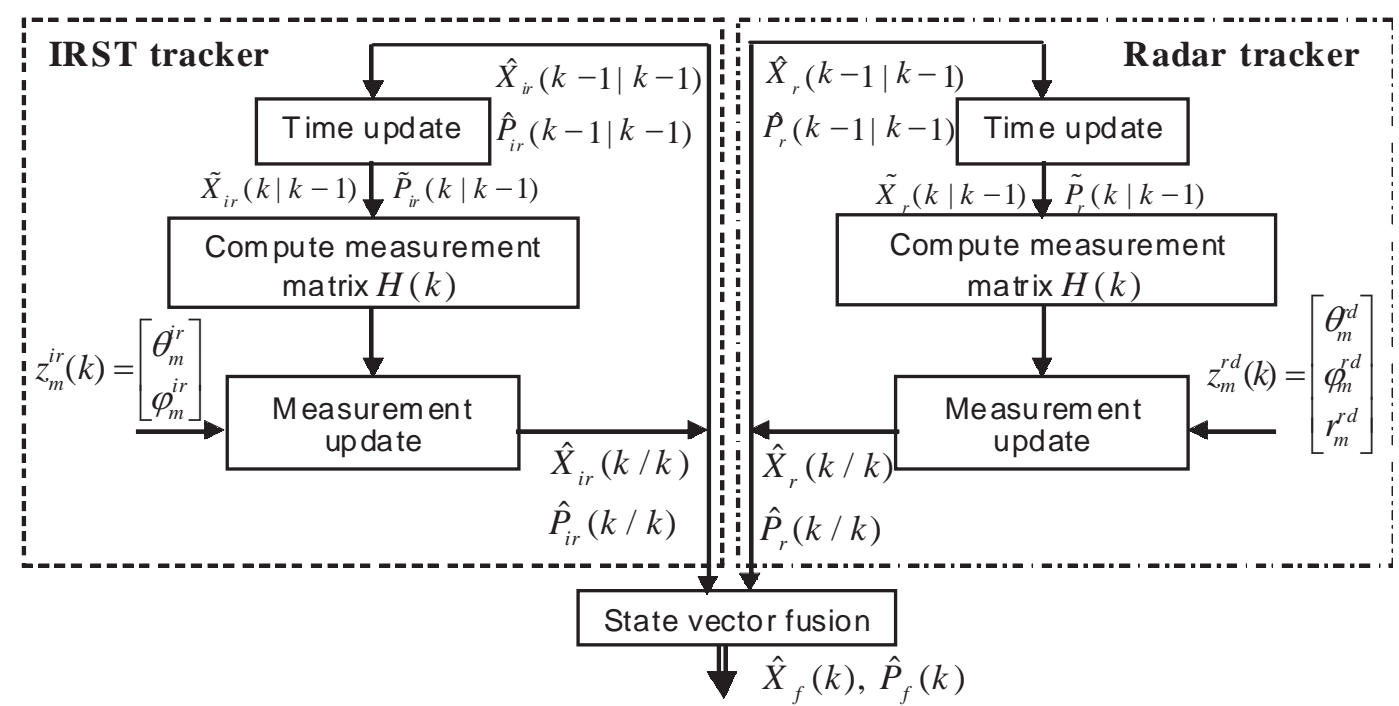

Figure 1. Information flow diagram of combining tracks obtained from radar and IRST.

needed to locate the position of the targets, track the targets as they move. Since estimation is to be done with the aid of sensors, appropriate sensors are chosen to collect data for different applications. Sensor mathematical models are required for generating realistic sensor data for validating estimation and tracking algorithms which could also be used for multi sensor data fusion (MSDF) applications. The estimation and tracking algorithms for real-time applications need to be validated using simulation studies. The mathematical models of the sensors help in generating realistic data. Radar and imaging sensor measurements are expressed in spherical coordinates. The following measurement models are used:

The radar measurements are:

$$
\begin{aligned}
& \text { range: } \quad r_{m}^{r d}(k)=r(k)+v_{r}^{r d}(k)(\mathrm{m}) \\
& \text { azimuth: } \theta_{m}^{r d}(k)=\theta(k)+v_{\theta}^{r d}(k)(\mathrm{rad}) \\
& \text { elevation: } \varphi_{m}^{r d}(k)=\varphi(k)+v_{\varphi}^{r d}(k)(\mathrm{rad})
\end{aligned}
$$

The IRST measurements are:

$$
\begin{aligned}
& \text { azimuth: } \theta_{m}^{i r}(k)=\theta(k)+v_{\theta}^{i r}(k)(\mathrm{rad}) \\
& \text { elevation: } \varphi_{m}^{i r}(k)=\varphi(k)+v_{\varphi}^{i r}(k)(\mathrm{rad})
\end{aligned}
$$

where superscripts $r d$ and $i r$ are used to indicate the radar and imaging sensor measurements, respectively. It is assumed that both the sensors are measuring the same target denoted by $(r(k), \theta(k), \varphi(k))$. The subscript $m$ indicates measurement and $v$ is the measurement noise added to simulate the realistic measurements.

\subsection{Target Motion Model}

The third order model, with position, velocity, and acceleration components in each of the three Cartesian coordinates $\mathrm{x}, \mathrm{y}$ and $\mathrm{z}$ has the following transition and process noise gain matrices:

$$
F=\operatorname{diag}\left[\begin{array}{lll}
\Phi & \Phi & \Phi
\end{array}\right] \text { and } G=\operatorname{diag}\left[\begin{array}{lll}
\varsigma & \varsigma & \varsigma
\end{array}\right]
$$

$$
\text { where } \Phi=\left[\begin{array}{ccc}
1 & T & T^{2} / 2 \\
0 & 1 & T \\
0 & 0 & 1
\end{array}\right] \text { and } \varsigma=\left[\begin{array}{c}
T^{3} / 6 \\
T^{2} / 2 \\
T
\end{array}\right]
$$

where $T$ is a sampling interval, $F$ is a state transition matrix, and $G$ is a process noise gain matrix.

\section{EXTENDED KALMAN FILTER}

Linear Kalman filter could be used for target tracking if the states and the measurements are in Cartesian coordinate system. Radar and IRST provide the measurements in a spherical coordinate system. In most of the cases, the state vector could be estimated in Cartesian coordinate system. Equation (1) is nonlinear and it needs to be linearised to fit into the Kalman filter framework entailing the use of extended Kalman filter (EKF).

\subsection{Time Propagation}

The state and state covariance matrices at time $k-1$ are predicted to time $k$ as follows:

$$
\begin{aligned}
& \tilde{X}(k+1 \mid k)=F \hat{X}(k \mid k) \\
& \tilde{P}(k+1 \mid k)=F \hat{P}(k \mid k) F^{T}+G Q G^{T}
\end{aligned}
$$

where $\hat{X}$ is the estimated state vector, $\hat{P}$ is the estimated state covariance matrix, $\tilde{X}$ is the predicted state, and $\tilde{P}$ is the predicted state covariance matrix.

\subsection{MEASUREMENT UPDATE}

Innovation: $\quad e=z_{m}(k+1)-\tilde{z}(k+1 \mid k)$

Innovation covariance:

$$
S=H(k) \tilde{P}(k+1 \mid k) H(k)^{T}+R
$$

where $\tilde{z}(k+1 \mid k)$ is the predicted measurement and $H(k)$ is the linearised measurement matrix. The measurement update part consists of the following equations. 
Filter gain: $K=\tilde{P}(k+1 \mid k) H(k)^{T} S^{-1}$

Updated state: $\hat{X}(k+1 \mid k+1)=\tilde{X}(k+1 \mid k)+K e$

Updated state covariance:

$$
\hat{P}(k+1 \mid k+1)=[I-K H(k)] \tilde{P}(k+1 \mid k)
$$

Predicted Measurement: Consider the state vector consisting of position, velocity and acceleration components in $\mathrm{x}-, \mathrm{y}$ - and $\mathrm{z}$-direction as:

$$
X=\left[\begin{array}{lllllllll}
x & \dot{x} & \ddot{x} & y & \dot{y} & \ddot{y} & z & \dot{z} & \ddot{z}
\end{array}\right]^{T}
$$

The predicted state is in the form:

$$
\left[\begin{array}{lllllllll}
\tilde{x} & \dot{\tilde{x}} & \ddot{\tilde{x}} & \tilde{y} & \dot{\tilde{y}} & \ddot{\tilde{y}} & \tilde{z} & \dot{\tilde{z}} & \ddot{\tilde{z}}
\end{array}\right]^{T}=\tilde{X}(k+1 \mid k)
$$

The predicted measurement of IRST is:

$$
\tilde{z}(k+1 \mid k)=h[\tilde{X}(k+1 \mid k)]=\left[\begin{array}{ll}
\tilde{\theta} & \tilde{\varphi}
\end{array}\right]^{T}
$$

The predicted measurement of radar is:

$$
\tilde{z}(k+1 \mid k)=h[\tilde{X}(k+1 \mid k)]=\left[\begin{array}{lll}
\tilde{\theta} & \tilde{\varphi} & \tilde{r}
\end{array}\right]^{T}
$$

Components in the predicted measurement are computed from the predicted state vector given in Eqn.(12).

$$
\tilde{\theta}=\tan ^{-1}\left(\frac{\tilde{y}}{\tilde{x}}\right) \tilde{\varphi}=\tan ^{-1}\left(\frac{\tilde{z}}{\sqrt{\tilde{x}^{2}+\tilde{y}^{2}}}\right) \tilde{r}=\sqrt{\tilde{x}^{2}+\tilde{y}^{2}+\tilde{z}^{2}}
$$

Linearised Measurement Matrix: Calculation of linearised measurement matrix can be accomplished by the finite difference method. This method is generalised and flexible ${ }^{4}$.

$$
H(k)=H_{i j}=\left.\frac{\partial h_{i}}{\partial x_{j}}\right|_{x=\tilde{X}(k \mid k-1)}=\frac{h_{i}\left(x_{j}+\Delta x_{j}\right)-h_{i}\left(x_{j}\right)}{\Delta x_{j}}
$$

where

$$
i=1,2, \ldots, \text { length of the measurement vector }
$$

$j=1,2, \ldots$, length of the state vector

$\Delta x_{j}=$ perturbation step size

For small perturbation $\Delta x$ in each of the unknown variables, the perturbed value $h_{i}\left(x_{j}+\Delta x_{j}\right)$ is computed. The corresponding elements of $H_{i j}$ are given by the finite difference in the function $h$ [Eqn (15)] to changes in that state. In general, a perturbation step size of $10^{-7}$ is considered to be adequate.

Nearest-neighbour extended Kalman filter (NNKF) is used to estimate the target state in the presence of clutter ${ }^{5}$.

\section{TRACK-TO-TRACK FUSION}

Consider the tracks coming from IRST and radar whose state estimations and covariance matrices are at scan $k$ as:

Track from IRST sensor: $\hat{X}_{1}(k \mid k), \hat{P}_{1}(k \mid k)$
Track from Radar: $\quad \hat{X}_{2}(k \mid k), \hat{P}_{2}(k \mid k)$

These two tracks are fused or combined using the following schemes.

\subsection{Fusion I}

In this fusion scheme, cross-covariance matrix is not considered in the fusion. Fused state vector and covariance matrix are ${ }^{5-7}$ :

$$
\begin{aligned}
X_{f}(k)= & \hat{X}_{1}(k \mid k)+\hat{P}_{1}(k \mid k)\left[\hat{P}_{1}(k \mid k)+\hat{P}_{2}(k \mid k)\right]^{-1} \\
& {\left[\hat{X}_{1}(k \mid k)+\hat{X}_{2}(k \mid k)\right] }
\end{aligned}
$$

equivalently

$$
\begin{aligned}
X_{f}(k)= & \hat{P}_{2}(k \mid k)\left[\hat{P}_{1}(k \mid k)+\hat{P}_{2}(k \mid k)\right]^{-1} \\
& \hat{X}_{1}(k \mid k)+\hat{P}_{1}(k \mid k) \\
& {\left[\hat{P}_{1}(k \mid k)+\hat{P}_{2}(k \mid k)\right]^{-1} \hat{X}_{2}(k \mid k) } \\
\hat{P}_{f}(k)= & \hat{P}_{1}(k \mid k)-\hat{P}_{1}(k \mid k)\left[\hat{P}_{1}(k \mid k)\right. \\
+ & \left.\hat{P}_{2}(k \mid k)\right]^{-1} \hat{P}_{1}^{T}(k \mid k)
\end{aligned}
$$

\subsection{Fusion II}

In this fusion scheme, cross-covariance matrix is considered in the fusion. The following computation has to be carried out during the time update. The predicted covariances of individual sensors and cross-covariance matrix between two sensors are computed as:

$$
\begin{aligned}
& \tilde{P}_{11}(k \mid k-1)=F \hat{P}_{11}(k-1 \mid k-1) F^{T}+G Q G^{T} \\
& \tilde{P}_{22}(k \mid k-1)=F \hat{P}_{22}(k-1 \mid k-1) F^{T}+G Q G^{T} \\
& \tilde{P}_{12}(k \mid k-1)=F \hat{P}_{12}(k-1 \mid k-1) F^{T}+G Q G^{T} \\
& \tilde{P}_{21}(k \mid k-1)=F \hat{P}_{21}(k-1 \mid k-1) F^{T}+G Q G^{T}
\end{aligned}
$$

These covariance and cross-covariance matrices are updated during measurement update stage. Covariance and Cross-covariance matrixes are updated as:

$$
\begin{aligned}
\hat{P}_{11}(k \mid k)= & \left(I-K_{1}(k) H_{1}(k)\right) \tilde{P}_{11}(k \mid k-1) \\
\hat{P}_{22}(k \mid k)= & \left(I-K_{2}(k) H_{2}(k)\right) \tilde{P}_{22}(k \mid k-1) \\
\hat{P}_{12}(k \mid k)= & {\left[I-K_{1}(k) H_{1}(k)\right] } \\
& \tilde{P}_{12}(k \mid k-1)\left[I-H_{2}^{T}(k) K_{2}^{T}(k)\right] \\
\hat{P}_{21}(k \mid k)= & {\left[I-K_{2}(k) H_{2}(k)\right] } \\
& \tilde{P}_{21}(k \mid k-1)\left[I-H_{1}^{T}(k) K_{1}^{T}(k)\right]
\end{aligned}
$$

where $K_{1}$ and $K_{2}$ are the Kalman gains from IRST and radar trackers respectively. $H_{1}$ and $H_{2}$ are the measurement matrices which are nonlinear functions of state vector from the IRST and radar trackers, respectively.

Then the fused state vector and covariance matrix are: 
Let

$$
\begin{aligned}
& A_{1}=\hat{P}_{11}(k \mid k)-\hat{P}_{12}(k \mid k) \\
& A_{2}=\hat{P}_{22}(k \mid k)-\hat{P}_{21}(k \mid k)
\end{aligned}
$$

The fusion covariance matrix can be computed as follows 6 .

$$
\begin{aligned}
P_{f}(k)= & P_{11}(k \mid k)-\left[P_{11}(k \mid k)-P_{c}(k \mid k)\right] \\
& P_{e}^{-1}(k)\left[P_{11}(k \mid k)-P_{c}(k \mid k)\right]^{T}
\end{aligned}
$$

where

$$
\begin{aligned}
& P_{e}(k)=P_{11}(k \mid k)+P_{22}(k \mid k)-P_{c}(k \mid k)-P_{c}(k \mid k)^{T} \\
& P_{c}(k \mid k)=\left(I-K_{1} H_{1}(k)\right) F P_{c}(k-1 \mid k-1) F^{T} \\
&\left(I-K_{2} H_{21}(k)\right)^{T}+\left(I-K_{1} H_{1}(k)\right) G Q G^{T} \\
&\left(I-K_{2} H_{2}(k)\right)^{T}
\end{aligned}
$$

with $\quad P_{c}(0 \mid 0)=0$

Using Eqn. (20) to Eqn.(27)

$$
\begin{aligned}
P_{c}(k \mid k)= & \left(I-K_{1} H_{1}(k)\right) F \hat{P}_{12}(k-1 \mid k-1) F^{T} \\
& \left(I-H_{2}(k) K_{2}\right)^{T}=\hat{P}_{12}(k \mid k) \\
P_{c}(k \mid k)^{T}= & \left(I-K_{2} H_{2}(k)\right) F \hat{P}_{21}(k-1 \mid k-1) F^{T} \\
& \left(I-H_{1}(k) K_{1}\right)^{T}=\hat{P}_{21}(k \mid k)
\end{aligned}
$$

Then Eqn. (30) can be written using the notation in Eqn. (28)

$$
\begin{aligned}
P_{e}(k \mid k)= & \hat{P}_{11}(k \mid k)+\hat{P}_{22}(k \mid k)-\hat{P}_{12}(k \mid k) \\
& -\hat{P}_{21}(k \mid k)=A_{1}+A_{2}
\end{aligned}
$$

Finally, the fused covariance matrix can be written as

$$
\begin{aligned}
P_{f}(k)= & \hat{P}_{11}(k \mid k)-\left[\hat{P}_{11}(k \mid k)-\hat{P}_{12}(k \mid k)\right] \\
& P_{e}^{-1}\left[\hat{P}_{11}(k \mid k)-\hat{P}_{12}(k \mid k)\right]^{T} \\
P_{f}(k)= & \hat{P}_{11}(k \mid k)-A_{1}\left[A_{1}+A_{2}\right]^{-1} A_{1}^{T}
\end{aligned}
$$

Fused state vector can be computed as

$$
\begin{aligned}
X_{f}(k)= & \hat{X}_{1}(k \mid k)+A_{1}\left[A_{1}+A_{2}\right]^{-1} \\
& {\left[\hat{X}_{2}(k \mid k)-\hat{X}_{1}(k \mid k)\right] }
\end{aligned}
$$

equivalently,

$$
\begin{gathered}
X_{f}(k)=A_{2}\left[A_{1}+A_{2}\right]^{-1} \hat{X}_{1}(k \mid k)+A_{1} \\
{\left[A_{1}+A_{2}\right]^{-1} \hat{X}_{2}(k \mid k)}
\end{gathered}
$$

\section{NUMERICAL SIMULATION}

The 3-DOF kinematic model, with position, velocity, and acceleration components in each of the three Cartesian coordinates $\mathrm{x}, \mathrm{y}$, and $\mathrm{z}$ has the transition and process noise gain matrices as Eqn. (4). The algorithm is validated using the simulated data. The simulation utilises the following parameters:

Sampling interval: $1 \mathrm{~s}$.

Process noise variance: 0.000012

Measurement noise variance:

Duration of simulation: $500 \mathrm{~s}$.

Initial state vector is:

$$
\begin{aligned}
{\left[\begin{array}{lllllllll}
x & \dot{x} & \ddot{x} & y & \dot{y} & \ddot{y} & z & \dot{z} & \ddot{z}
\end{array}\right] } \\
\quad=\left[\begin{array}{lllllllll}
100 & -0.2 & -0.05 & 100 & -2 & 0.01 & 100 & -0.5 & 0.1
\end{array}\right]
\end{aligned}
$$

The simulated true and noisy measurements of IRST and radar are shown in Fig. 2. The initial state vector is given by:

$$
\hat{X}_{0}=0.9 X_{t}
$$

where $\hat{X}_{0}$ is initial estimated state vector at scan number one, and $X_{t}$ is true state vector at scan number one.

\begin{tabular}{lccc}
\hline Sensor & $\begin{array}{c}\text { Azimuth } \\
(\mathbf{r a d})\end{array}$ & $\begin{array}{c}\text { Elevation } \\
(\mathbf{r a d})\end{array}$ & $\begin{array}{c}\text { Range } \\
(\mathbf{m})\end{array}$ \\
\hline IRST & $10^{-6}$ & $10^{-6}$ & -- \\
Radar & $10^{-2}$ & $10^{-2}$ & 5 \\
\hline
\end{tabular}

In general, the initial states are chosen based on the first few measurements. Rule of thumb is that with good instrumentation, the expected errors would not be $>10$ per cent. However, the errors are also accounted for by choosing an appropriate $\hat{P}_{0}$. In this simulated example, the value of is chosen as above and it was found that for this case, other initial conditions gave similar convergence results.

The expression for the initial state error covariance matrix is given by

$$
\hat{P}_{0}=\operatorname{diag}\left[\left(X_{t}-\hat{X}_{0}\right)^{2}\right]
$$

The filter performance is checked by computing 5

(a) The percentage fit error (PFE) in $x, y$, and $z$ positions:

$\operatorname{PFE} x=100 * \frac{\operatorname{norm}\left(x_{t}-\hat{x}\right)}{\operatorname{norm}\left(x_{t}\right)}$

similarly for $y$ and $z$ positions

(b) Root mean square error in position:

$\mathrm{RSSPE}=\sqrt{\frac{1}{N} \sum_{i=1}^{N} \frac{\left(x_{i}-\hat{x}_{i}\right)^{2}+\left(y_{i}-\hat{y}_{i}\right)^{2}+\left(z_{i}-\hat{z}_{i}\right)^{2}}{3}}$

Similarly for velocity (RMSVE) and acceleration (RMSAE)

(c) Root sum square error in position:

$$
\operatorname{RSSPE}=\sqrt{(x-\hat{x})^{2}+(y-\hat{y})^{2}+(z-\hat{z})^{2}}
$$

Similarly for velocity (RSSVE) and acceleration (RSSAE) (d) Mean absolute error (MAE) in positions:

$\operatorname{MAE} x=\frac{1}{N} \sum_{i=1}^{N}\left|x_{t}(i)-\hat{x}(i)\right|$

Similarly for $y$ and $z$ positions, velocities and accelerations 

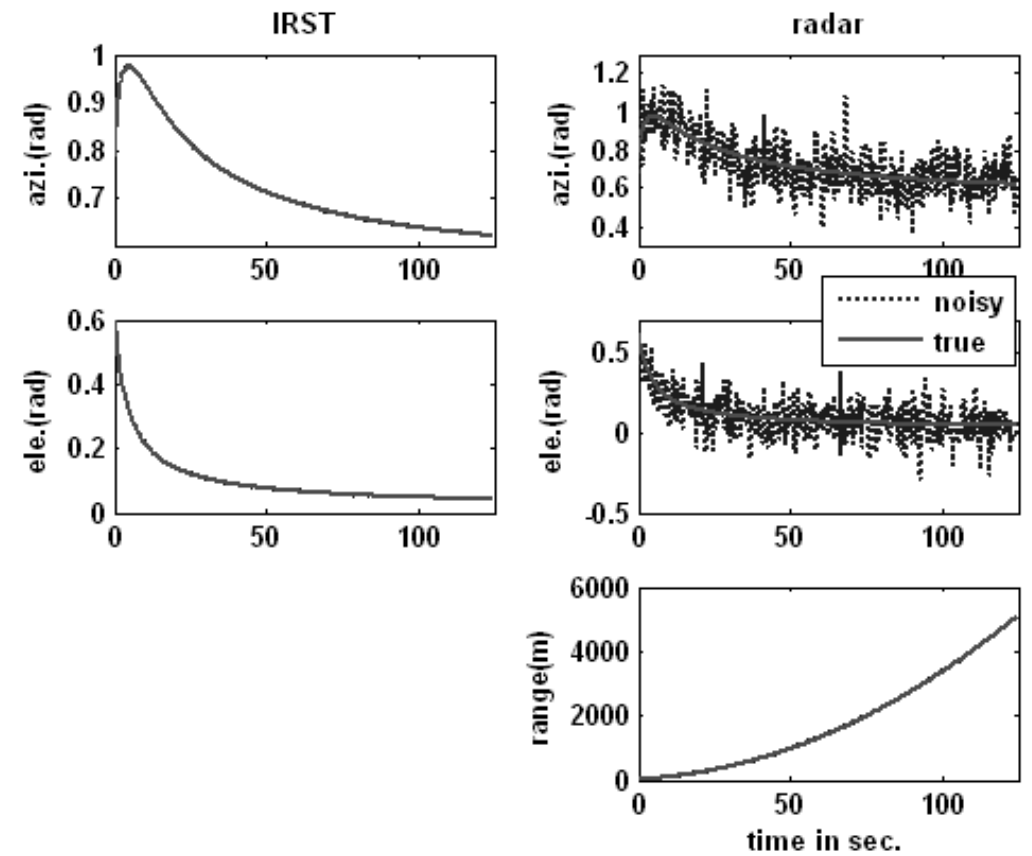

Figure 2. IRST and Radar measurements in polar coordinates.

(e) Absolute error (AE) in $x, y$, and $z$ positions:

$$
\operatorname{AE} x(i)=\left|x_{t}(i)-\hat{x}(i)\right| \quad i=1,2, \ldots, N
$$

Similarly for $y$ and $z$ positions, velocities and accelerations

(f) State error $(X-\hat{X})$ with the theoretical bounds of $\pm 2 \sqrt{\hat{P}_{(k, k)}}$

(g) Innovation sequence $(z(k)-\tilde{z}(k \mid k-1))$ with theoretical

$$
\text { bounds of } \pm 2 \sqrt{S_{(k, k)}}
$$

Performance of the proposed algorithm is evaluated using seventy five Monte Carlo simulations. The percentage fit error (PFE) and mean absolute error in $\mathrm{x}_{-}, \mathrm{y}-$ and $\mathrm{z}$ positions are shown in Table 1.

The RMSPE, RMSVE and RMSAE are shown in Table 2. It is also observed that after fusion the PFE is comparatively very less which indicates the true and estimated positions

Table 1. PFE and MAE in $x-, y-$ and z-positions

\begin{tabular}{lcccccc}
\hline & PFEx & PFEy & PFEz & MAEx & MAEy & MAEz \\
\hline IRST & 9.07 & 9.07 & 9.07 & 130 & 100 & 8.94 \\
Radar & 0.36 & 0.6 & 4.21 & 5.29 & 6.36 & 4.09 \\
Fusion1 & 0.05 & 0.08 & 0.33 & 0.67 & 0.76 & 0.26 \\
Fusion2 & 0.05 & 0.07 & 0.32 & 0.66 & 0.75 & 0.25 \\
\hline
\end{tabular}

are well matched. It is also observed that both fusion I and fusion II give almost similar results for this particular scenario, hence the rest of the results are presented from fusion II. The percentage of improvement using the fusion algorithm in terms of percentage fit error, absolute error and root mean square error in position, velocity and acceleration are shown in Table 3 . It shows clearly that the necessity of fusion at final target state estimation.

The true and estimated $z$ positions are shown in Fig. 3. The fused trajectories are well matched with the true trajectories of the individual trackers. The estimated trajectory by IRST sensor is deviated from the true. It could be due to unavailability of range information in measurements. The estimated trajectory by radar follows the true but not as much as the fused trajectory. The root sum square error in position and acceleration are shown in Figs 4(a) and 4(b) respectively. It is observed that after fusion these errors are comparatively very less and the estimated trajectory follows the true trajectory. The state

Table 2. RMSPE, RMSVE and RMSAE

\begin{tabular}{llll}
\hline & RMSPE & RMSVE & RMSAE \\
\hline IRST & 123.99 & 2.415 & 0.031 \\
Radar & 7 & 0.247 & 0.108 \\
fusion1 & 0.853 & 0.108 & 0.009 \\
fusion2 & 0.843 & 0.103 & 0.009 \\
\hline
\end{tabular}

Table 3. Percentage improvement using fusion of IRST and radar trackers

\begin{tabular}{lccccccccc}
\hline With & \multicolumn{3}{c}{ In terms of PFE } & \multicolumn{3}{c}{ In terms of MAE } & \multicolumn{3}{c}{ In terms of RMS error } \\
\cline { 2 - 10 } & $\mathbf{x}(\boldsymbol{\%})$ & $\mathbf{y}(\boldsymbol{\%})$ & $\mathbf{z}(\boldsymbol{\%})$ & $\mathbf{x}(\boldsymbol{\%})$ & $\mathbf{y}(\boldsymbol{\%})$ & $\mathbf{z}(\boldsymbol{\%})$ & $\begin{array}{r}\text { RMSPE } \\
(\boldsymbol{\%})\end{array}$ & $\begin{array}{r}\text { RMSVE } \\
(\boldsymbol{\%})\end{array}$ & $\begin{array}{r}\text { RMSAE } \\
(\boldsymbol{\%})\end{array}$ \\
\hline IRST & 99.4 & 99.11 & 96.36 & 99.96 & 99.24 & 96.31 & 99.31 & 95.74 & 70.97 \\
Radar & 86.11 & 86.67 & 92.16 & 87.33 & 88.05 & 93.64 & 78.81 & 58.3 & 91.67 \\
\hline
\end{tabular}




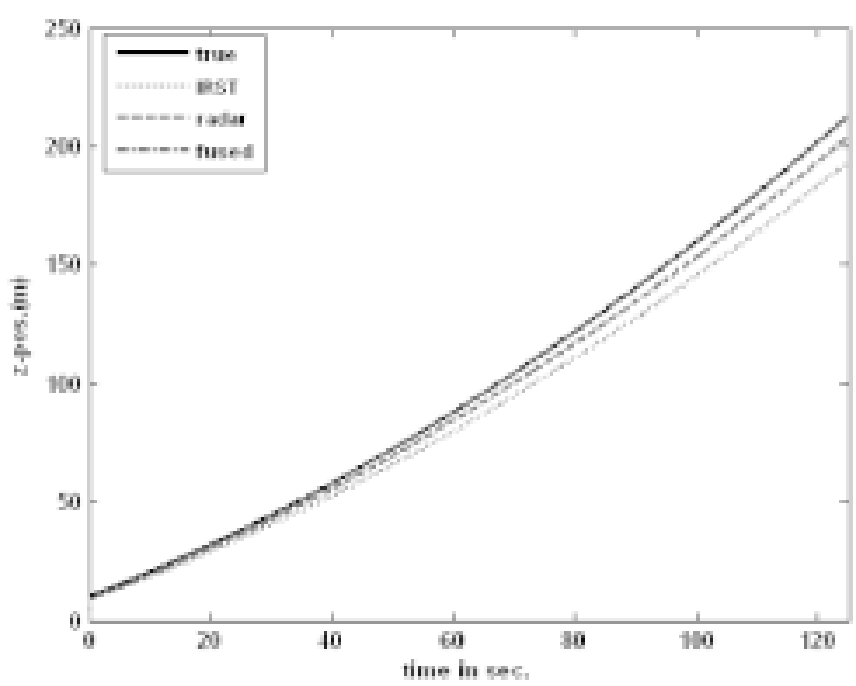

Figure 3. True, estimated and fused z-positions.

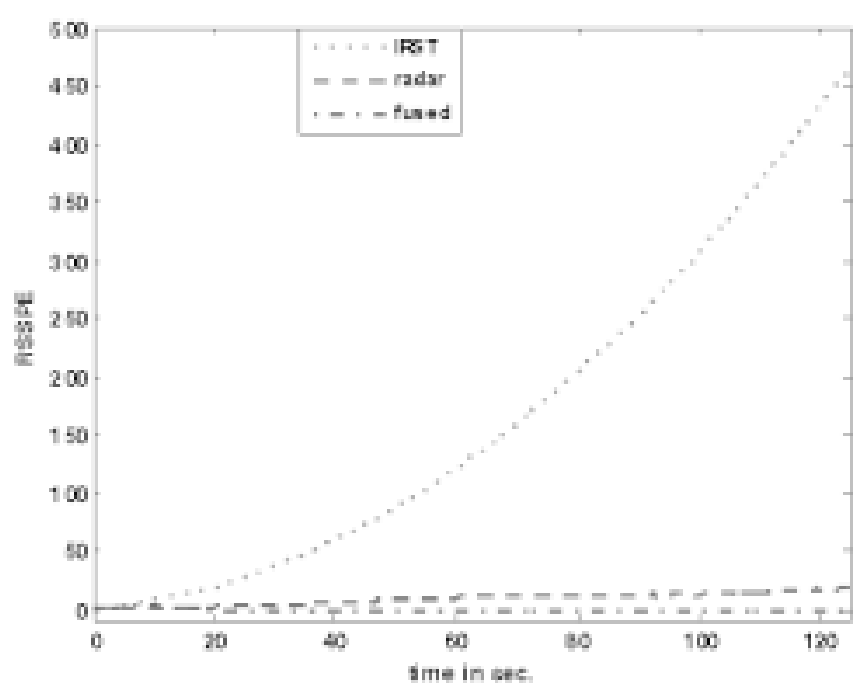

Figure 4 (a). Root sum square error in position.

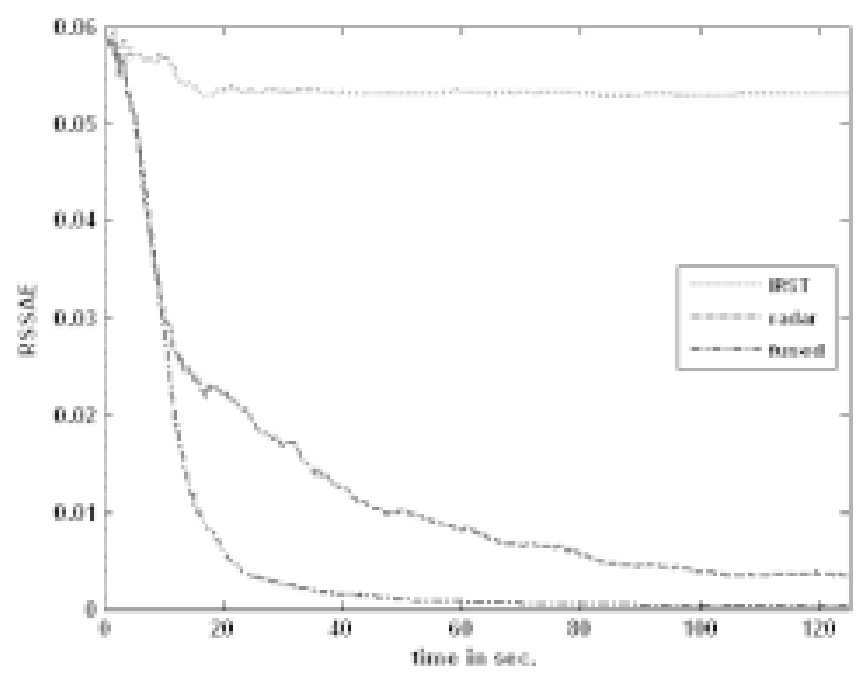

Figure 4(b). Root sum square error in acceleration.

errors in position and acceleration along with their theoretical bounds are shown in Figs 5(a) and 5(b), respectively. After fusion the uncertainty in estimation is comparatively reduced.
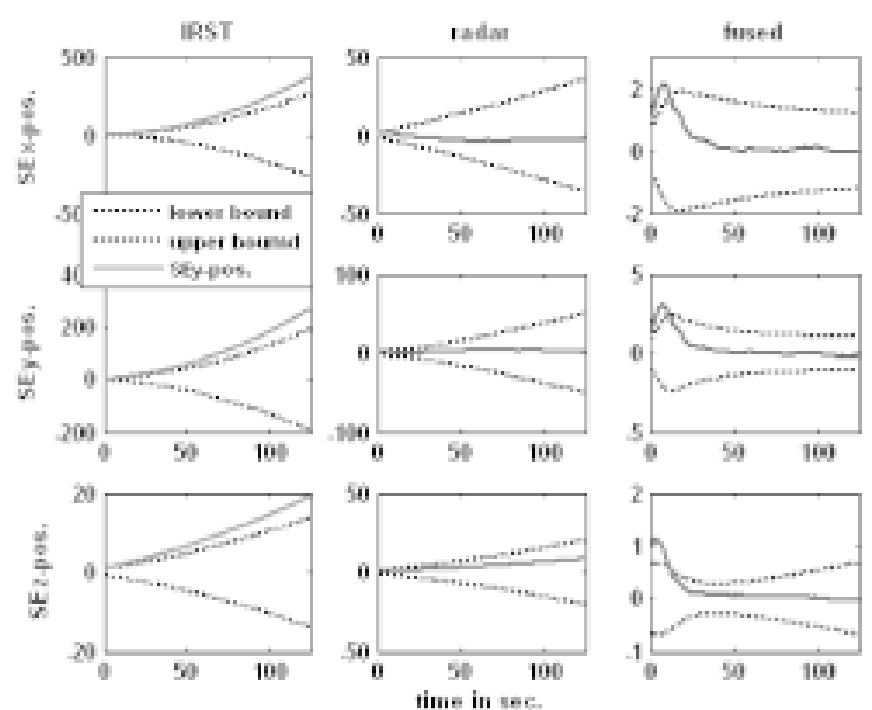

Figure 5(a). Position state error with theoretical bounds.
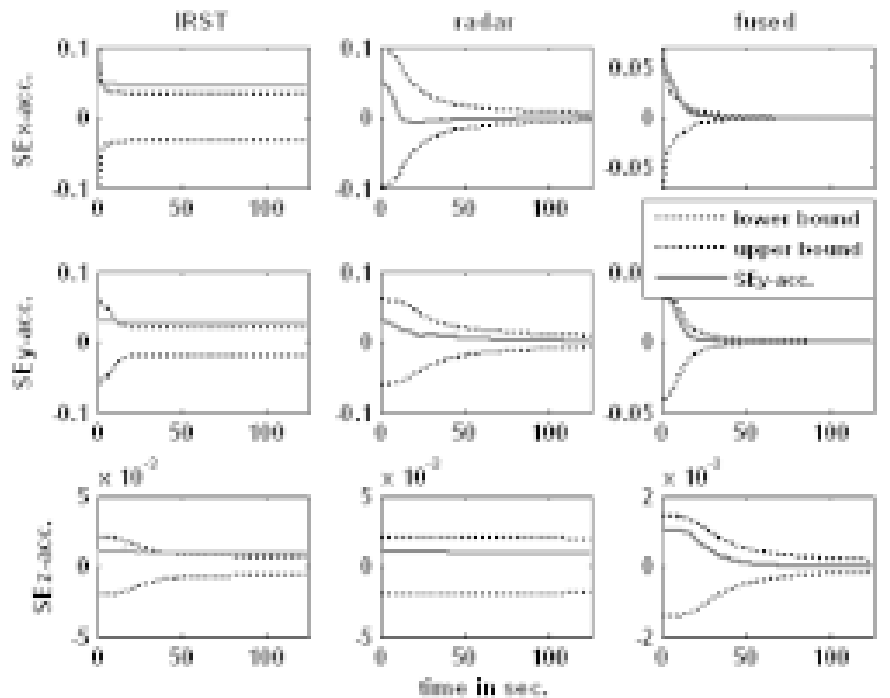

Figure 5(b). Acceleration state error with theoretical bounds.

This could be the key outcome from the fusion. The innovation sequence along with their theoretical bounds for both IRST and radar trackers are shown in Fig. 6. The innovation is within the bounds which indicate that all the information in the data is extracted by the filters. Absolute error in $x$-, $y$ and $z$-acceleration are shown in Fig. 7. The errors are less in all axes with fusion algorithm.

Clutter was added to the noisy measurements to test the robustness of the algorithm. Measurements of IRST and radar along with clutter are shown in Fig. 8. Similar observation was made in the presence of clutter as shown in Figs 9 to 11 and Tables 4 and 5. One can see the effect of clutter in Fig. 11, where the innovation is out of theoretical bounds when there is no valid measurement and the state estimation is only based on state prediction.

\section{CONCLUSION}

Tracking algorithms for IRST and radar are implemented and their performance are checked with simulated data. 

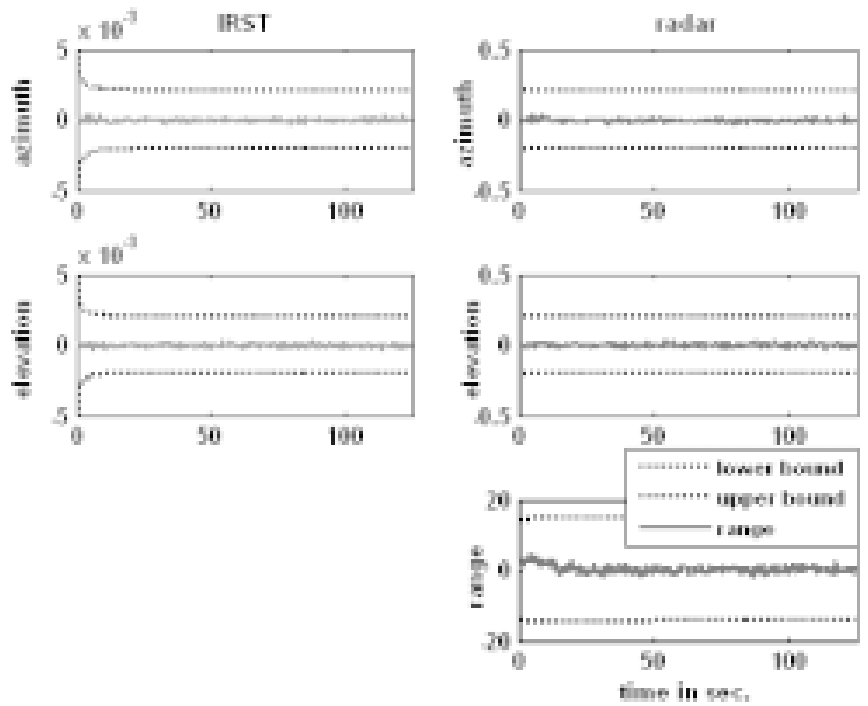

Figure 6. Innovation sequence with theoretical bounds.
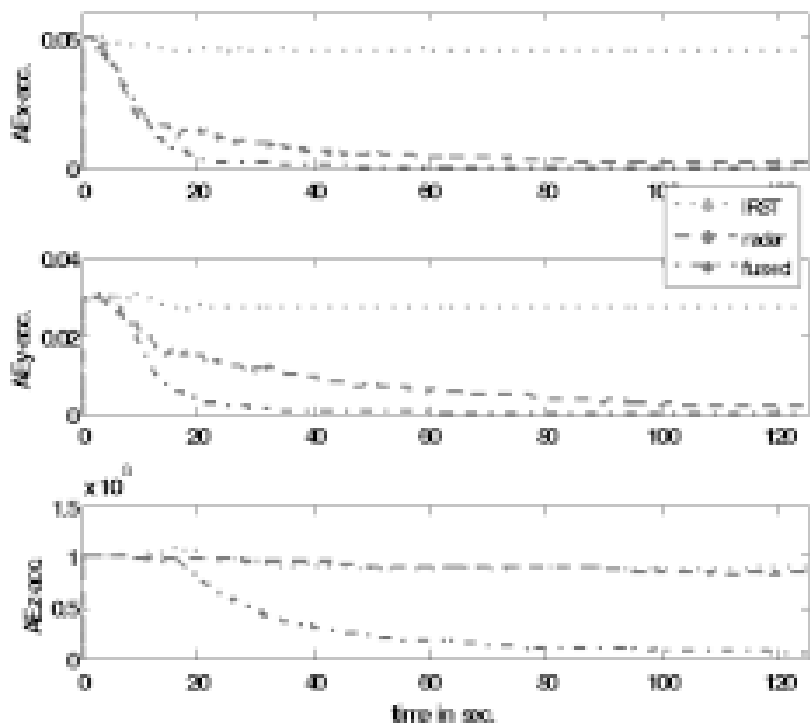

Figure 7. Absolute error in $x$-, $y$ - and z-acceleration.
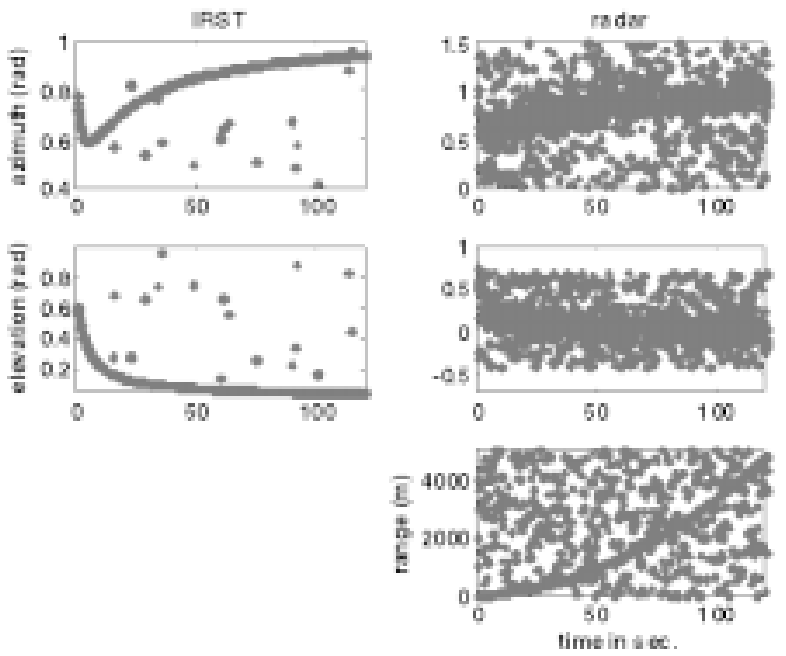

Figure 8. IRST and radar measurements including clutter in polar coordinates.

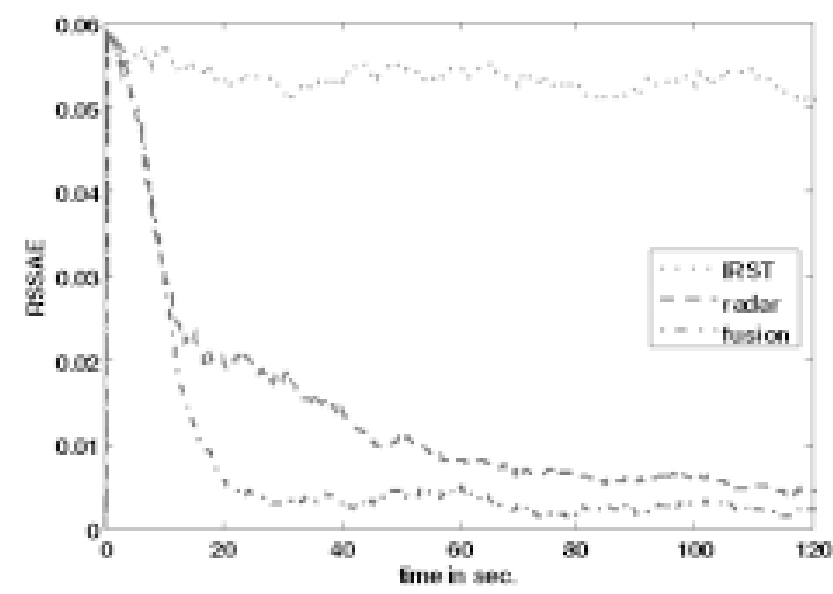

Figure 9. Root sum square error in acceleration in the presence of clutter.
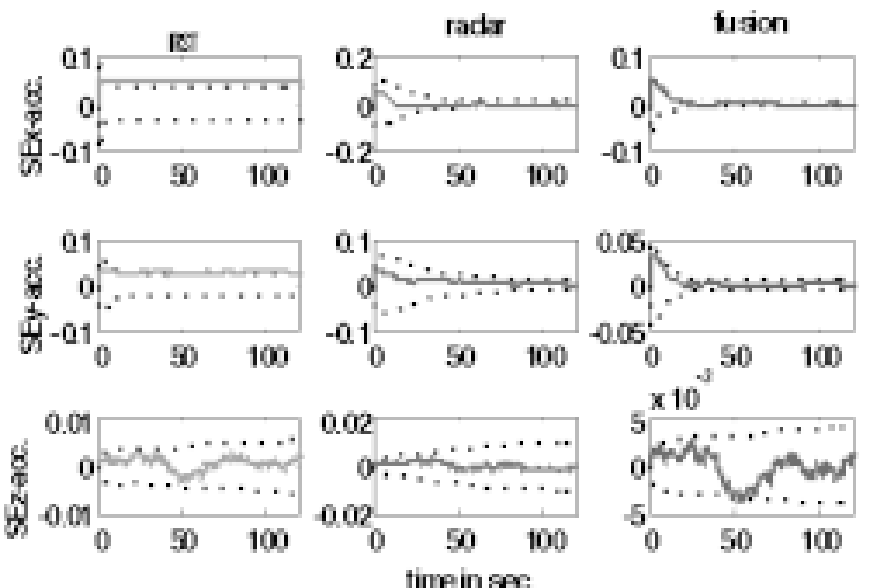

Figure 10. Acceleration state error with theoretical bounds in presence of clutter.
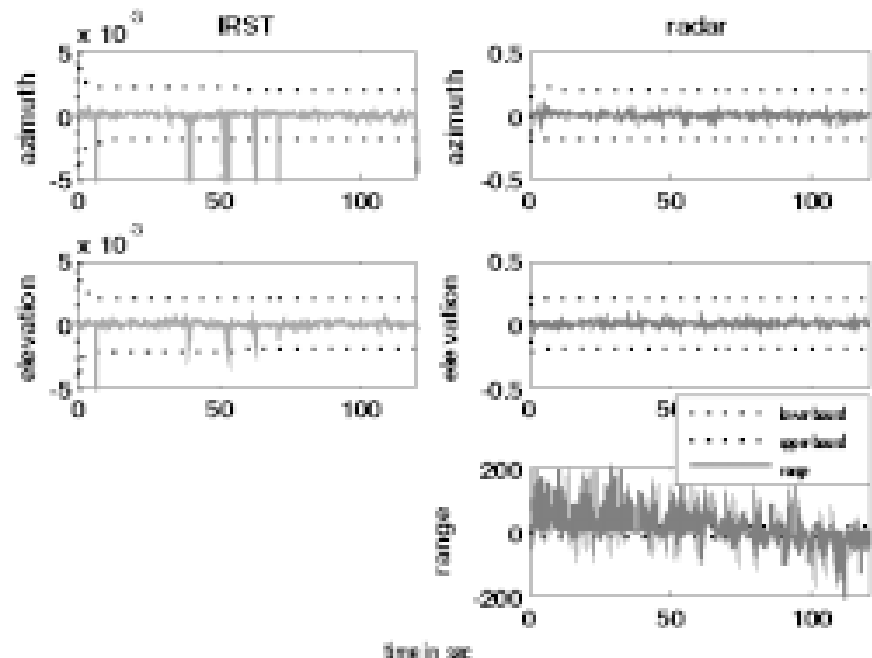

Figure 11. Innovation sequence with theoretical bounds in the presence of clutter. 
Table 4. PFE and MAE in $x-, y$ - and z-positions in the presence of clutter

\begin{tabular}{lcccccr}
\hline & PFEx & PFEy & PFEz & MAEx & MAEy & MAE \\
\hline IRST & 8.94 & 8.95 & 8.92 & 128.91 & 98.96 & 8.89 \\
Radar & 0.38 & 0.65 & 4.12 & 5.68 & 6.87 & 3.97 \\
Fusion II & 0.057 & 0.09 & 0.42 & 0.85 & 0.86 & 0.36 \\
\hline
\end{tabular}

Table 5. RMSPE, RMSVE and RMSAE in the presence of clutter

\begin{tabular}{llll}
\hline & RMSPE & RMSVE & RMSAE \\
\hline IRST & 122.082 & 2.392 & 0.031 \\
Radar & 7.385 & 0.258 & 0.011 \\
Fusion II & 0.973 & 0.115 & 0.009 \\
\hline
\end{tabular}

Detailed mathematical expressions are given which could be useful for implementation. Performance evaluation metrices are presented to check the tracking algorithm performance. Two fusion schemes have been presented and evaluated. It is concluded that both schemes performed alike with the fusion scheme II gives slightly better results. The robustness of the algorithm was tested with clutter added to the noisy measurements. From the results, it is also concluded that fusion of IRST and radar would improve the tracking performance and reduce the positional uncertainty compared to individual trackers.

\section{REFERENCES}

1. Blackman, Samuel \& Popoli, Robert. Design and analysis of modern tracking systems. Artech House, London, 1999.

2. Hall, David, L. \& McMullen, Sonya A. H. Mathematical techniques in multisensor data fusion. Ed. 2, Artech House, London, 2004.
3. Bar-Shalam, Yaakov \& Li, X. Estimation and tracking: Principles, techniques, and softwares. Artech House, London, 1993.

4. Girija, G. \& Zorn, Christoph. Flight path reconstruction for sensor failure detection and health monitoring. report No. NAL PD FC 0418, 2004.

5. Naidu, V.P.S.; Girija, G. \& Raol, J.R. Data association and fusion algorithms for tracking in the presence of measurement loss. J. Inst. Engs., (I), 2005, (86)5, 17 28.

6. Saha, R.K. Effect of common process noise on twosensor track fusion. J. Gui. and Dyn., 1996, (19)4, 829-35.

7. Naidu, V.P.S. \& Raol, J.R. Fusion of radar and infrared search and track data using Kalman filter. Report No. NAL PD FC 0517, 2005.

\section{Contributor}

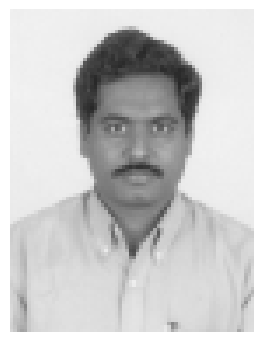

Mr V.P.S. Naidu obtained his ME in Medical electronics from Anna University, Chennai, in 1997. He is working as a scientist at National Aerospace Laboratories, Bangalore, since December 2001 in the area of multi sensor data fusion and target tracking. His areas of interest are image registration, tracking, and data fusion. 